\title{
Where Is the Lightest Charmed Scalar Meson?
}

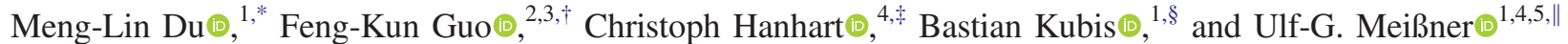 \\ ${ }^{1}$ Helmholtz-Institut für Strahlen- und Kernphysik and Bethe Center for Theoretical Physics, \\ Universität Bonn, D-53115 Bonn, Germany \\ ${ }^{2}$ CAS Key Laboratory of Theoretical Physics, Institute of Theoretical Physics, Chinese Academy of Sciences, \\ Beijing 100190, China \\ ${ }^{3}$ School of Physical Sciences, University of Chinese Academy of Sciences, Beijing 100049, China \\ ${ }^{4}$ Institute for Advanced Simulation, Institut für Kernphysik and Jülich Center for Hadron Physics, \\ Forschungszentrum Jülich, D-52425 Jülich, Germany \\ ${ }^{5}$ Tbilisi State University, 0186 Tbilisi, Georgia
}

(Received 16 December 2020; revised 18 March 2021; accepted 7 April 2021; published 13 May 2021)

\begin{abstract}
The lightest charmed scalar meson is known as the $D_{0}^{*}(2300)$, which is one of the earliest new hadron resonances observed at modern $B$ factories. We show here that the parameters assigned to the lightest scalar $D$ meson are in conflict with the precise LHCb data of the decay $B^{-} \rightarrow D^{+} \pi^{-} \pi^{-}$. On the contrary, these data can be well described by an unitarized chiral amplitude containing a much lighter charmed scalar meson, the $D_{0}^{*}(2100)$. We also extract the low-energy $S$-wave $D \pi$ phase of the decay $B^{-} \rightarrow D^{+} \pi^{-} \pi^{-}$from the data in a model-independent way, and show that its difference from the $D \pi$ scattering phase shift can be traced back to an intermediate $\rho^{-}$exchange. Our work highlights that an analysis of data consistent with chiral symmetry, unitarity, and analyticity is mandatory in order to extract the properties of the ground-state scalar mesons in the singly heavy sector correctly, in analogy to the light scalar mesons $f_{0}(500)$ and $K_{0}^{*}(700)$.
\end{abstract}

DOI: 10.1103/PhysRevLett.126.192001

Introduction.-Since the discovery of the $D_{s 0}^{*}(2317)$ [1], many hadrons were observed beyond the quark model expectations, which have seriously challenged the understanding of the hadron spectrum in terms of the conventional quark model that identifies mesons as $\bar{q} q$ states. The observation that the $D_{s 0}^{*}(2317)$ [1] and $D_{s 1}(2460)$ [2] are significantly lighter than expected by the quark model, around 2.48 and $2.55 \mathrm{GeV}$ [3-5], has driven the development of various models, including $D^{(*)} K$ hadronic molecules [6-13], tetraquark states [14,15], and mixtures of $c \bar{q}$ with tetraquarks [16]. In 2004, two new charm-nonstrange structures, the $D_{0}^{*}(2300)[17,18]$, called $D_{0}^{*}(2400)$ previously, and $D_{1}(2430)$ [17], were reported as the SU(3) partners of the $D_{s 0}^{*}(2317)$ and $D_{s 1}(2460)$, respectively. The observations posed a puzzle: why are the masses of the two nonstrange mesons, $D_{0}^{*}(2300)$ and $D_{1}(2430)$, almost equal to their strange siblings, i.e., the $D_{s 0}^{*}(2317)$ and $D_{s 1}(2460)$ ? Thanks to new data from both lattice quantum chromodynamics (QCD) [19-24] and the LHCb experiment [25], it was recently demonstrated that the various puzzles in the charm meson spectrum can be solved

Published by the American Physical Society under the terms of the Creative Commons Attribution 4.0 International license. Further distribution of this work must maintain attribution to the author(s) and the published article's title, journal citation, and DOI. Funded by SCOAP . naturally in the framework of unitarized chiral perturbation theory (UChPT) [26-28] that allows one to calculate the nonperturbative dynamics of Goldstone bosons scattering off the $D_{(s)}^{(*)}$ mesons in a controlled way. The combination of UChPT and lattice QCD not only reproduced the correct $D_{s 0}^{*}(2317)$ mass [19], but also predicted its pion mass dependence $[23,29]$. The solution provided for the $\mathrm{SU}(3)$ mass hierarchy puzzle mentioned above is that instead of only one heavy state, $D_{0}^{*}(2300)$ in the channel $(S, I) \equiv($ strangeness, isospin $)=(0,1 / 2)$, there are two states, one lighter and one heavier $[9,11,13,19,26,30$ 34]. The most recent studies revealed their pole locations to be at $\left(2105_{-8}^{+6}-i 102_{-11}^{+10}\right)$ and $\left(2451_{-26}^{+35}-i 134_{-8}^{+7}\right) \mathrm{MeV}$ $[26,27]$, respectively. The $\mathrm{SU}(3)$ partner of the $D_{s 0}^{*}(2317)$ is the lighter one, denoted as $D_{0}^{*}(2100)$ in the following, which restores the expected mass hierarchy. The heavier pole on the other hand is a member of a different multiplet. Support for the presence of two poles comes from an analysis of the high-quality LHCb data on the decays $B^{-} \rightarrow$ $D^{+} \pi^{-} \pi^{-}$[25], $B_{s}^{0} \rightarrow \bar{D}^{0} K^{-} \pi^{+}$[35], $B^{0} \rightarrow \bar{D}^{0} \pi^{-} \pi^{+}$[36], $B^{-} \rightarrow D^{+} \pi^{-} K^{-}$[37], and $B^{0} \rightarrow \bar{D}^{0} \pi^{-} K^{+}$[38] performed in Refs. [27,39], as well as from the fact that their existence is consistent with the lattice energy levels [19-23] for the relevant two-body scattering $[26,32,40]$. This two-pole structure indeed emerges as a more general pattern in the hadron spectrum, see, e.g., Ref. [41]. 
Despite the phenomenological success of this picture in describing the available lattice and LHCb data, the observation that the lightest $D_{0}^{*}$ has a mass around $2.1 \mathrm{GeV}$ has not entered the Review of Particle Physics (RPP) [42] yet, which still lists the $D_{0}^{*}(2300)$ as the lightest charmed scalar meson and the $D_{1}(2430)$ as the corresponding axial-vector meson. In this Letter, we demonstrate that the $D_{0}^{*}(2300)$ as in the RPP is not consistent with the most precise data for $B^{-} \rightarrow D^{+} \pi^{-} \pi^{-}$, contrary to the $D_{0}^{*}(2100)$ predicted in UChPT, and conclude that the positive-parity charmnonstrange meson spectrum in the RPP needs to be revised.

$D \pi S$-wave phase of $B^{-} \rightarrow D^{+} \pi^{-} \pi^{-}$. - The decay amplitude in the low-energy region of the $D \pi$ system can be decomposed into $S, P$, and $D$ waves,

$$
\mathcal{A}_{B^{-} \rightarrow D^{+} \pi^{-} \pi^{-}}(s, z)=\sum_{\ell=0}^{2} \sqrt{2 \ell+1} \mathcal{A}_{\ell}(s) P_{\ell}\left(z_{s}\right),
$$

where $\mathcal{A}_{\ell}(s)$ with $\ell=0,1,2$ correspond to the amplitudes with $D^{+} \pi^{-}$in the $S, P$, and $D$ waves, respectively, $s$ is the c.m. energy squared of the $D^{+} \pi^{-}$system, and $P_{\ell}\left(z_{s}\right)$ are the Legendre polynomials with $z_{s}$ the cosine of the helicity angle of the $D^{+} \pi^{-}$system, i.e., the angle between the moving directions of the two pions in the $D^{+} \pi^{-}$c.m. frame. The angular moments are determined by weighting the data with the Legendre polynomials $P_{\ell}(z)$ [25]. They contain contributions from certain partial waves and their interference terms, and thus the corresponding phase variations. The first few moments are given by $[25,27,39]$

$$
\begin{aligned}
\left\langle P_{0}\right\rangle & \propto\left|\mathcal{A}_{0}\right|^{2}+\left|\mathcal{A}_{1}\right|^{2}+\left|\mathcal{A}_{2}\right|^{2}, \\
\left\langle P_{2}\right\rangle & \propto \frac{2}{5}\left|\mathcal{A}_{1}\right|^{2}+\frac{2}{7}\left|\mathcal{A}_{2}\right|^{2}+\frac{2}{\sqrt{5}}\left|\mathcal{A}_{0}\right|\left|\mathcal{A}_{2}\right| \cos \left(\delta_{2}-\delta_{0}\right), \\
\left\langle P_{13}\right\rangle & \equiv\left\langle P_{1}\right\rangle-\frac{14}{9}\left\langle P_{3}\right\rangle \propto \frac{2}{\sqrt{3}}\left|\mathcal{A}_{0}\right|\left|\mathcal{A}_{1}\right| \cos \left(\delta_{1}-\delta_{0}\right),
\end{aligned}
$$

with $\delta_{i}$ the phase of $\mathcal{A}_{i}$, i.e., $\mathcal{A}_{i}=\left|\mathcal{A}_{i}\right| e^{i \delta_{i}}$. As first proposed in Ref. [27], we use the linear combination $\left\langle P_{13}\right\rangle$ instead of $\left\langle P_{1}\right\rangle$ and $\left\langle P_{3}\right\rangle$ individually, since it only depends on the $S-P$-wave interference up to $\ell=2$ and is particularly sensitive to the $S$-wave phase motion.

For $M_{D^{+} \pi^{-}}<2.2 \mathrm{GeV}$, even the $D$ wave can be neglected, since the narrow tensor resonance $D_{s 2}(2460)$ is sufficiently far away. This can be verified from the data of the angular moments, i.e., Fig. 3 in Ref. [25]. Therefore, in this kinematic regime one obtains

$$
\cos \left(\delta_{0}-\delta_{1}\right)=\sqrt{\frac{3}{10}} \frac{\left\langle P_{13}\right\rangle}{\sqrt{\left\langle P_{2}\right\rangle} \sqrt{\left\langle P_{0}\right\rangle-\frac{5}{2}\left\langle P_{2}\right\rangle}}
$$

for the $S-P$ phase difference. The $P$ wave is dominated by the vector resonance $D^{*}(2007)^{0}$ below the $D^{+} \pi^{-}$threshold

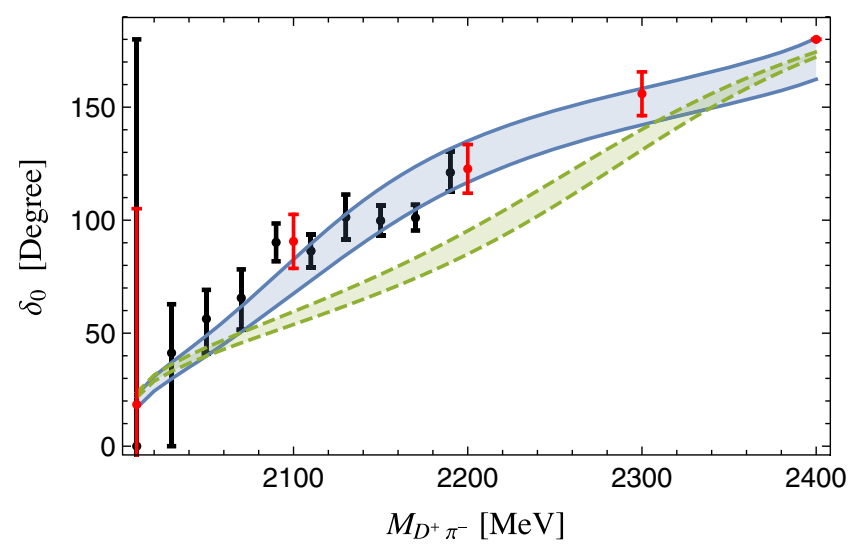

FIG. 1. Comparison of the predictions of Eq. (5) from UChPT (blue) and a Breit-Wigner parametrization (green) for $\delta_{0}$ with the phase extracted in Ref. [25] (red) and that using Eq. (3) (black). The bands correspond to errors propagated from the input UChPT scattering amplitudes and from the Breit-Wigner resonance parameters.

with a width of less than $60 \mathrm{keV}[43,44]$. The next vector $D^{*}$ resonance is far above this energy region. Thus, the phase of the $P$ wave $\delta_{1}$ can be safely fixed to $180^{\circ}$ for the region we are interested in. The $S$-wave $D \pi$ phase motion below $2.2 \mathrm{GeV}$ can then be extracted, see Fig. 1. For comparison, the phase motion of the $D^{+} \pi^{-} S$ wave up to $2.4 \mathrm{GeV}$ obtained in the LHCb analysis [25] (with the phase at $2.4 \mathrm{GeV}$ fixed to $180^{\circ}$ ) is also shown, which is fully in line with the phase we extracted from Eq. (3) below $2.2 \mathrm{GeV}$.

For $M_{D^{+} \pi^{-}}<2.4 \mathrm{GeV}$, the effect of the $\rho$ meson could be significant via the coupled channel $B^{-} \rightarrow D^{0} \pi^{0} \pi^{-}$; see Fig. 2. This follows directly from the large branching ratio $\mathcal{B}\left(B^{-} \rightarrow D^{0} \rho^{-}\right)=1.34 \%$, which is an order larger than $\mathcal{B}\left(B^{-} \rightarrow D^{+} \pi^{-} \pi^{-}\right)=0.107 \%$ [42]. It is therefore reasonable to assume that the decay $B^{-} \rightarrow D^{+} \pi^{-} \pi^{-}$is dominated by the process $B^{-} \rightarrow D^{0} \rho^{-} \rightarrow D^{0} \pi^{0} \pi^{-} \rightarrow D^{+} \pi^{-} \pi^{-}$. By virtue of soft-pion theorems, one has [45]

$$
\begin{aligned}
& \left.\mathcal{A}\left(B^{-} \rightarrow D^{+} \pi^{-} \pi^{-}\right)\right|_{p_{\pi^{-}} \rightarrow 0}=\frac{1}{F_{\pi}} \mathcal{A}\left(B^{0} \rightarrow \bar{D}^{0} \pi^{0}\right), \\
& \left.\mathcal{A}\left(B^{-} \rightarrow D^{0} \pi^{0} \pi^{-}\right)\right|_{p_{\pi^{0}} \rightarrow 0}=-\frac{1}{F_{\pi}} \mathcal{A}\left(B^{-} \rightarrow D^{0} \pi^{-}\right),
\end{aligned}
$$

where $p_{\pi^{-}\left(\pi^{0}\right)}$ is the momentum of the $\pi^{-}\left(\pi^{0}\right)$, and $F_{\pi}$ is the pion decay constant (in the chiral limit). From $\mathcal{B}\left(B^{0} \rightarrow\right.$ $\left.\bar{D}^{0} \pi^{0}\right)=2.63 \times 10^{-4}$ and $\mathcal{B}\left(B^{-} \rightarrow D^{0} \pi^{-}\right)=4.68 \times 10^{-3}$, one concludes that at low energies for the $D^{+} \pi^{-}\left(D^{0} \pi^{0}\right)$ system, the amplitude of $B^{-} \rightarrow D^{0} \pi^{0} \pi^{-}$is much larger than that of $B^{-} \rightarrow D^{+} \pi^{-} \pi^{-}$. Furthermore, isospin symmetry shows that for the decays $B \rightarrow D \pi \pi$ with even relative angular momenta between the pions, the amplitude for $B^{-} \rightarrow D^{+} \pi^{-} \pi^{-}$is larger than that of $B^{-} \rightarrow D^{0} \pi^{0} \pi^{-}$by a factor of $2 \sqrt{2}[27,39,46,47]$. As in addition even angular 


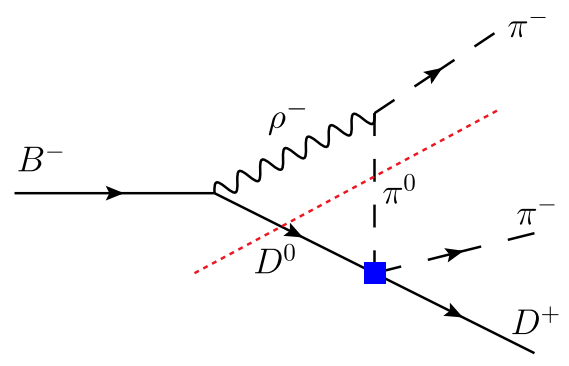

FIG. 2. The decay $B^{-} \rightarrow D^{+} \pi^{-} \pi^{-}$via the coupled channel $B^{-} \rightarrow D^{0} \pi^{0} \pi^{-}$. The filled square denotes the $D^{0} \pi^{0} \rightarrow D^{+} \pi^{-} T$ matrix element.

momenta here imply isospin $I=2$ and therefore nonresonant partial waves, the relative angular momentum of $\pi^{0} \pi^{-}$ in the decay $B^{-} \rightarrow D^{0} \pi^{0} \pi^{-}$is by far dominantly odd in the low-energy regime for $D^{0} \pi^{0}$, and the $\rho^{-}$plays a crucial role.

If we assume that the decay $B^{-} \rightarrow D^{+} \pi^{-} \pi^{-}$is dominated by the process in Fig. 2, the $D \pi S$-wave part of the triangle diagram can be estimated by the integral

$$
\mathcal{A}_{0}^{\mathrm{trig}}(s)=\frac{1}{\pi} \int_{s_{\mathrm{th}}}^{\infty} d s^{\prime} \frac{\hat{P}\left(s^{\prime}\right) \rho\left(s^{\prime}\right) T_{D^{0} \pi^{0} \rightarrow D^{+} \pi^{-}}\left(s^{\prime}\right)}{s^{\prime}-s},
$$

where $\hat{P}(s)$ is the production amplitude for $B^{-} \rightarrow D^{0} \rho^{-} \rightarrow$ $D^{0} \pi^{0} \pi^{-}$projected to the $D^{0} \pi^{0} s$ channel, $\rho(s)=$ $\sqrt{\lambda\left(s, M_{D}^{2}, M_{\pi}^{2}\right)} /(16 \pi s)$ is the $D \pi$ phase space with $\lambda(a, b, c)=a^{2}+b^{2}+c^{2}-2 a b-2 a c-2 b c$ the Källén function, $T_{D^{0} \pi^{0} \rightarrow D^{+} \pi^{-}}(s)$ the $S$-wave scattering amplitude for $D^{0} \pi^{0} \rightarrow D^{+} \pi^{-}$, and $s_{\mathrm{th}}=\left(M_{D}+M_{\pi}\right)^{2}$. The expression for $\hat{P}(s)$ is the same as $\hat{\mathcal{F}}_{0}^{1 / 2}(s)$ in Eq. (12) below.

The evaluation of Eq. (5) depends on the asymptotic behavior of the integrand, which is divergent in general. We may estimate Eq. (5) using a cutoff at $\sqrt{s_{\max }}=\sqrt{q_{\max }^{2}+M_{D}^{2}}+\sqrt{q_{\max }^{2}+M_{\pi}^{2}}$, where $q_{\max } \approx$ $1 \mathrm{GeV}$ (another way is to introduce a form factor, e.g., $e^{-\left(s-s_{\text {th }}\right) / s_{0}}$ with $\left.s_{0}=\mathcal{O}(1 \mathrm{GeV})[48]\right)$. We evaluate Eq. (5) by employing both the $D \pi$ scattering amplitude from UChPT [19] and that of a Breit-Wigner (BW) parametrization of the $D_{0}^{*}(2300)$ for comparison, despite the deficiencies of the latter discussed in Ref. [39]; see also Ref. [49].

The results with $q_{\max }=1 \mathrm{GeV}$ are shown in Fig. 1, where the solid blue band and the green dashed band correspond to the $D \pi$ scattering amplitudes from UChPT and BW, respectively. The obtained phase describes the data perfectly for the UChPT amplitude, while the BW one fails. We have checked that the obtained phases are insensitive to a variation of the cutoff in a reasonable region, $q_{\max } \in[0.8,1.2] \mathrm{GeV}$.

Khuri-Treiman formalism.-While Eq. (5) provides a reasonable estimation of the $S$-wave decay amplitude with a clear underlying physical picture, it does not respect three-body unitarity. In order to check if the conclusion formulated above is robust, we cure this deficiency by employing the Khuri-Treiman equations [50], which are based on two-body elastic phase shifts and explicitly generate the crossed-channel rescattering between final-state particles. The formulas are constructed from dispersion relations for the related crossed scattering processes and then analytically continued to the decay region, referring to the continuation of the triangle graph [51].

We can write amplitudes for $\mathcal{A}_{+--}\left(B^{-} \rightarrow D^{+} \pi^{-} \pi^{-}\right)$and $\mathcal{A}_{00-}\left(B^{-} \rightarrow D^{0} \pi^{0} \pi^{-}\right)$in terms of single-variable functions according to a reconstruction theorem [47,52],

$$
\begin{aligned}
\mathcal{A}_{+--}(s, t, u)= & \mathcal{F}_{0}^{1 / 2}(s)+\frac{\kappa(s)}{4} z_{s} \mathcal{F}_{1}^{1 / 2}(s) \\
& +\frac{\kappa(s)^{2}}{16}\left(3 z_{s}^{2}-1\right) \mathcal{F}_{2}^{1 / 2}(s)+(t \leftrightarrow s), \\
\mathcal{A}_{00-}(s, t, u)= & -\frac{1}{\sqrt{2}} \mathcal{F}_{0}^{1 / 2}(s)-\frac{\kappa(s)}{4 \sqrt{2}} z_{s} \mathcal{F}_{1}^{1 / 2}(s) \\
& -\frac{\kappa(s)^{2}}{16 \sqrt{2}}\left(3 z_{s}^{2}-1\right) \mathcal{F}_{2}^{1 / 2}(s)+\frac{\kappa_{u}(u)}{4} z_{u} \mathcal{F}_{1}^{1}(u),
\end{aligned}
$$

where the subindex $\ell$ and superindex $I$ of the singlevariable amplitudes $\mathcal{F}_{\ell}^{I}$ represent the angular momentum and isospin, respectively, and only the $I<3 / 2$ and $\ell \leq 2$ terms are taken into account. The Mandelstam variables of the $B$-meson decay $B^{-}\left(p_{B}\right) \rightarrow D\left(p_{D}\right) \pi\left(p_{1}\right) \pi^{-}\left(p_{2}\right)$ are $s=\left(p_{B}-p_{2}\right)^{2}, \quad t=\left(p_{B}-p_{1}\right)^{2}$, and $u=\left(p_{B}-p_{D}\right)^{2}$. The corresponding angles are given by

$z_{s} \equiv \cos \theta_{s}=\frac{s(t-u)-\Delta}{\kappa(s)}, \quad z_{u} \equiv \cos \theta_{u}=\frac{t-s}{\kappa_{u}(u)}$,

where $\quad \kappa(s)=\lambda^{1 / 2}\left(s, M_{D}^{2}, M_{\pi}^{2}\right) \lambda^{1 / 2}\left(s, M_{B}^{2}, M_{\pi}^{2}\right), \quad \kappa_{u}(u)=$ $\lambda^{1 / 2}\left(u, M_{B}^{2}, M_{D}^{2}\right) \sqrt{1-4 M_{\pi}^{2} / u}, \quad$ and $\quad \Delta=\left(M_{B}^{2}-M_{\pi}^{2}\right) \times$ $\left(M_{D}^{2}-M_{\pi}^{2}\right)$.

Since we are interested in the $s$-channel process, we use the index $A(B)$ to label the two-body channels corresponding to $D^{+} \pi^{-}$and $D^{0} \pi^{0}$. The partial-wave decomposition for the decay amplitudes $\mathcal{A}_{A}$ reads

$$
\mathcal{A}_{A}\left(s, z_{s}\right)=\sum_{I, \ell} b_{I, \ell}^{A} P_{\ell}\left(z_{s}\right) f_{\ell}^{I}(s),
$$

with $b_{I, \ell}^{A}$ denoting Clebsch-Gordan coefficients. By comparing with Eq. (1), it is easy to obtain $\mathcal{A}_{\ell}(s)=$ $(2 \ell+1)^{-1 / 2} \sum_{I} b_{I, \ell}^{1} f_{\ell}^{I}(s)$. We have the following partialwave unitarity relation for elastic rescattering:

$$
\operatorname{disc} f_{\ell}^{I}(s)=2 i f_{\ell}^{I}(s) \sin \delta_{\ell}^{I}(s) e^{-i \delta_{\ell}^{I}(s)} \theta\left(s-s_{\mathrm{th}}\right),
$$

where $\delta_{t}^{I}(s)$ is the elastic final-state scattering phase shift. The discontinuities of $f_{\ell}^{I}$ and those of the single-variable 
functions $\kappa^{\ell} \mathcal{F}_{\ell}^{I}$ coincide on the right-hand cut by construction. Thus, one has

$\operatorname{disc} \mathcal{F}_{\ell}^{I}(s)=2 i\left[\mathcal{F}_{\ell}^{I}(s)+\hat{\mathcal{F}}_{\ell}^{I}(s)\right] \sin \delta_{\ell}^{I}(s) e^{-i \delta_{\ell}^{I}(s)} \theta\left(s-s_{\mathrm{th}}\right)$,

where the inhomogeneities $\hat{\mathcal{F}}_{\ell}^{I}(s)$ encode the left-hand cut contributions and are free of discontinuities on the righthand cut. This discontinuity relation is solved by

$\mathcal{F}_{\ell}^{I}(s)=\Omega_{\ell}^{I}(s)\left\{Q_{\ell}^{I}(s)+\frac{s^{n}}{\pi} \int_{s_{\mathrm{th}}}^{\infty} \frac{d s^{\prime}}{s^{\prime n}} \frac{\sin \delta_{\ell}^{I}\left(s^{\prime}\right) \hat{\mathcal{F}}_{\ell}^{I}\left(s^{\prime}\right)}{\left|\Omega_{\ell}^{I}\left(s^{\prime}\right)\right|\left(s^{\prime}-s\right)}\right\}$,

where $\Omega_{\ell}^{I}(s)=\exp \left\{s / \pi \int_{s_{\mathrm{th}}}^{\infty} d s^{\prime} \delta_{\ell}^{I}\left(s^{\prime}\right) /\left[s^{\prime}\left(s^{\prime}-s\right)\right]\right\}$ is the Omnès function [53], $Q_{\ell}^{I}(s)$ is a polynomial at least of degree $(n-1)$ (see discussion below), and the number of subtractions $n$ is chosen to guarantee the convergence of the dispersion integral.

The inhomogeneity $\hat{\mathcal{F}}_{\ell}^{I}$ is determined by the partialwave decomposition of Eq. (6) as the projection of the crossed-channel amplitudes onto the considered channel. Around the $D \pi$ threshold in the $s$ channel, $\sqrt{t} \sim 5 \mathrm{GeV}$, there is no resonance in the $t$ channel, and thus the interaction is supposed to be very weak. The only possible significant crossed-channel effect is from the $\rho$ meson through $B^{-} \rightarrow D^{0} \pi^{0} \pi^{-}$. The resulting inhomogeneity for the $S$-wave $s$-channel amplitude is [47]

$$
\hat{\mathcal{F}}_{0}^{1 / 2}(s)=-\frac{1}{4 \sqrt{2}} \int_{-1}^{1} d z_{s}(t-s) \mathcal{F}_{1}^{1}(u) .
$$

For technical details regarding this integral, see Ref. [54] and the Supplemental Material [55].

The full solution for the decay amplitudes can be obtained by solving a set of coupled integral equations in terms of a few linearly independent complex subtraction constants contained in $Q_{\ell}^{I}(s)$, which cannot be determined a priori in the framework of dispersion theory. Since we are only interested in the $D \pi$ low-energy regime and especially in its $S$ wave, based on the large branching ratio of $B^{-} \rightarrow D^{0} \rho^{-}$, it is reasonable to approximate $\mathcal{F}_{1}^{1}(u)$ in Eq. (6) by a $\mathrm{BW}$ function for the $\rho$ meson. In this case, $\mathcal{F}_{1}^{1}(u)$ behaves as $u^{-1}$ for $u \rightarrow \infty$, thus $\hat{\mathcal{F}}_{\ell}^{I}(s)$ in Eq. (12) approaches a constant as $s \rightarrow \infty$. The number of the subtractions $n$ in Eq. (11) is then determined by the asymptotic behavior of the scattering phase $\delta_{\ell}^{I}(s)$. For the BW phase and that of UChPT taken from Ref. [19], one single subtraction is sufficient. Moreover, the phase of UChPT, as well as that of the BW, is unreliable at high energies. Thus, the dispersion integral will be evaluated up to a cutoff $\Lambda$, and the effect of cutting off the integral may be absorbed into the polynomial $Q_{\ell}^{I}(s)$. Explicitly, for an integral

$$
\begin{aligned}
g(s) & =\int_{s_{\mathrm{th}}}^{\infty} d s^{\prime} \frac{f\left(s^{\prime}\right)}{s^{\prime}-s}=\int_{s_{\mathrm{th}}}^{\Lambda} d s^{\prime} \frac{f\left(s^{\prime}\right)}{s^{\prime}-s}+\int_{\Lambda}^{\infty} d s^{\prime} \frac{f\left(s^{\prime}\right)}{s^{\prime}-s} \\
& \approx g_{0}+g_{1} s+\int_{s_{\mathrm{th}}}^{\Lambda} d s^{\prime} \frac{f\left(s^{\prime}\right)}{s^{\prime}-s} .
\end{aligned}
$$

For simplicity, we neglect the $I=3 / 2$ contribution since it contains no resonances. Therefore, for the $S$-wave amplitude at low energies, Eq. (11) can be written as

$$
\begin{aligned}
\mathcal{F}_{0}^{1 / 2}(s)= & \Omega_{0}^{1 / 2}(s)\left\{g_{0}+g_{1} \frac{s-M_{D}^{2}}{M_{D}^{2}}\right. \\
& \left.+\frac{s}{\pi} \int_{s_{\text {th }}}^{\Lambda} \frac{d s^{\prime}}{s^{\prime}} \frac{\sin \delta_{0}^{1 / 2}\left(s^{\prime}\right) \hat{\mathcal{F}}_{0}^{1 / 2}\left(s^{\prime}\right)}{\left|\Omega_{0}^{1 / 2}\left(s^{\prime}\right)\right|\left(s^{\prime}-s\right)}\right\} .
\end{aligned}
$$

The constants $g_{0}$ and $g_{1}$ have to be fixed by data.

We fit $\left\langle P_{0}\right\rangle,\left\langle P_{13}\right\rangle$, and $\left\langle P_{2}\right\rangle$ of the decay $B^{-} \rightarrow$ $D^{+} \pi^{-} \pi^{-}$up to $2.4 \mathrm{GeV}$, which is below the $D \eta$ and $D_{s} \bar{K}$ thresholds. To describe the angular moments, one needs the explicit amplitudes for the $D \pi P$ and $D$ waves. The $P$ wave can be safely parametrized as $\delta_{1}^{1 / 2}(s)=$ $\pi \theta\left(s-M_{D^{* 0}}^{2}\right)$ as discussed below Eq. (3). Consequently, the dispersion integral (11) for the $P$ wave can be neglected since $\sin \pi=0$. The $D$ wave is dominated by the resonance $D_{2}^{0}(2460)$ with a width of $47.5 \mathrm{MeV}$ [42], which is above the region we are interested in. Thus, the $D$-wave phase is close to 0 below $2.4 \mathrm{GeV}$, and the corresponding dispersion integral can be neglected as well. Therefore, for the $P$ - $\left(D\right.$-)wave amplitudes $\mathcal{F}_{1,2}^{1 / 2}$, we can use the same BW forms as those in the LHCb analysis [25], which is equivalent to the corresponding Omnès function multiplied by a polynomial. In the isobar model used in Ref. [25], complex factors are introduced for each resonance BW function. Without crossed-channel effects, these factors become real according to Watson's theorem [56]. For the $P$ and $D$ waves, as discussed above, the Omnès representation should be a good approximation in the energy region we are interested in, and the normalization factor is real. We also consider a complex normalization and find the results unchanged.

For the $D \pi S$ wave, we employ both the scattering phase shifts from UChPT [19], which contains the $D_{0}^{*}(2100)$, and the BW for the $D_{0}^{*}(2300)$. The fit results are shown in Fig. 3, where the blue and green bands correspond to the best fits from UChPT and the BW, respectively. While UChPT describes the data very well with $\chi^{2} /$ d.o.f. $=1.2$, the BW fails to reproduce the data with $\chi^{2} /$ d.o.f. $=2.0$. The difference of these two values is significant from the statistical point of view: the corresponding $p$ values are 0.1 for the UChPT fit and $3 \times 10^{-5}$ for the BW fit, respectively. Thus, the former can be accepted as a good description of the data, while the latter is highly disfavored [57]. The error bands correspond to the $1 \sigma$ uncertainties propagated from 

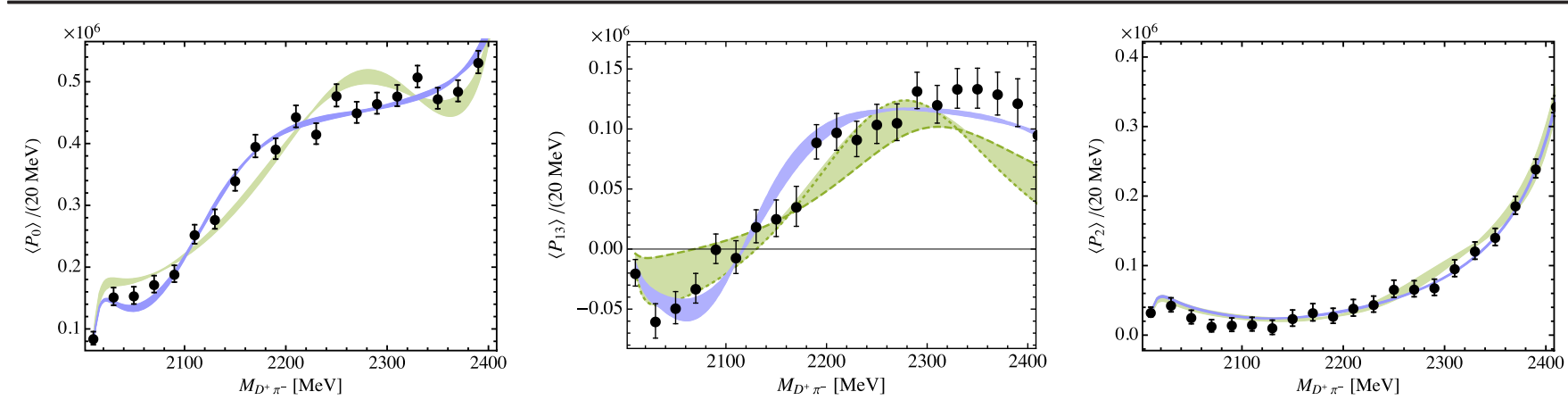

FIG. 3. Results of UChPT (blue) and the BW description (green), with the best fits $\chi^{2} /$ d.o.f. $=1.2$ and 2.0 , respectively.

the input phases. The borders of the band of $\left\langle P_{13}\right\rangle$ for the BW are plotted in dotted and dashed curves to make it evident that the data for $\left\langle P_{0}\right\rangle$ and $\left\langle P_{13}\right\rangle$ cannot be described by the BW phase. With the fitted parameters $g_{0}$ and $g_{1}$, we obtain the $S$-wave phase of the decay amplitude for $B^{-} \rightarrow$ $D^{+} \pi^{-} \pi^{-}$shown in Fig. 4, where the results corresponding to UChPT and the BW are plotted as blue and green bands, respectively. As expected, UChPT describes the $S$-wave phase extracted using Eq. (3) and that obtained in Ref. [25] well up to $2.4 \mathrm{GeV}$. For the BW one, although the error band is broad, either the low-energy or the high-energy region cannot be described.

Conclusion.-The existence of the $D_{0}^{*}(2300)$ as given in the RPP is the starting point of many theoretical analyses (see, e.g., Refs. [58-61]). The results obtained in this Letter show that the $D_{0}^{*}(2300)$, whose resonance parameters were obtained using the BW parametrization from the Belle [17] and $B A B A R$ [62] analyses, is in conflict with the much more precise LHCb data for $B^{-} \rightarrow D^{+} \pi^{-} \pi^{-}$, which, however, can be well reproduced by the UChPT amplitude containing the $D_{0}^{*}(2100)$.

We expect that the $D_{1}(2430)$ as given in the RPP [42] will also be in conflict with future high-quality data of $B^{-} \rightarrow D^{*+} \pi^{-} \pi^{-}$from LHCb [63] and Belle-II, and that the

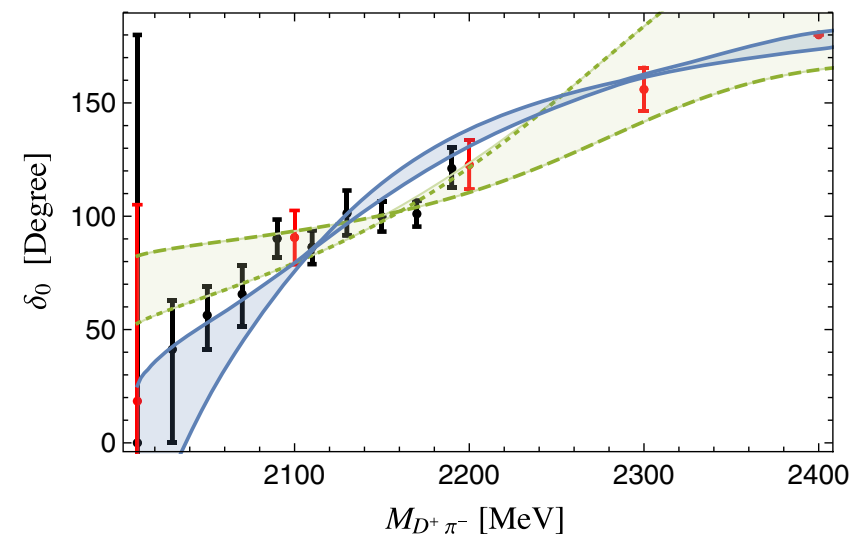

FIG. 4. Phases obtained from Eq. (14) with scattering phase shifts from UChPT (blue) and BW (green). lightest $D_{1}$ meson is the $D_{1}(2250)$ predicted by UChPT [26,27].

The $D_{0}^{*}$ is analogous to the more famous $f_{0}(500)$ and $K_{0}^{*}(700)$, whose masses have been significantly shifted from earlier versions of the RPP due to improved data and improved theoretical analyses-for recent discussions see Refs. [64-68] and the review on scalar mesons in the RPP [42]. We expect a similar change in all systems emerging from the scattering of a pion off an isospin-nonsinglet hadron. The lightest resonance in that case should not be extracted from data using the usual BW form-a parametrization accounting for chiral symmetry and coupled channels is mandatory. The $D \pi S$ wave phase extracted model independently here provides valuable information for further understanding matter-field-Goldstone-boson scattering and the structure of positive-parity heavy hadrons.

This work is supported in part by the National Natural Science Foundation of China (NSFC) under Grants No. 11835015, No. 12047503, and No. 11961141012, by the NSFC and the Deutsche Forschungsgemeinschaft (DFG, German Research Foundation) through the funds provided to the Sino-German Collaborative Research Center "Symmetries and the Emergence of Structure in QCD" (NSFC Grant No. 12070131001, DFG Project-ID 196253076-TRR110), by the Chinese Academy of Sciences (CAS) under Grants No. XDB34030000 and No. QYZDB-SSW-SYS013, and by the CAS Center for Excellence in Particle Physics (CCEPP). The work of U. G. M. was also supported by the Chinese Academy of Sciences (CAS) President's International Fellowship Initiative (PIFI) (Grant No. 2018DM0034), by VolkswagenStiftung (Grant No. 93562), and by the EU (Strong2020).

Note added.-Recently, a lattice calculation also concluded that the $D_{0}^{*}$ mass should be lower than the RPP value [69]. The authors found a mass of $(2196 \pm 64) \mathrm{MeV}$ with a pion mass of $239 \mathrm{MeV}$, only $(77 \pm 64) \mathrm{MeV}$ above the $D \pi$ threshold. Thus, our conclusion receives a strong support from lattice QCD calculations. 
*du@hiskp.uni-bonn.de

†fkguo@itp.ac.cn

*c.hanhart@fz-juelich.de

§ubis@hiskp.uni-bonn.de

"meissner@hiskp.uni-bonn.de

[1] B. Aubert et al. (BABAR Collaboration), Phys. Rev. Lett. 90, 242001 (2003).

[2] D. Besson et al. (CLEO Collaboration), Phys. Rev. D 68, 032002 (2003); 75, 119908(E) (2007).

[3] S. Godfrey and N. Isgur, Phys. Rev. D 32, 189 (1985).

[4] S. Godfrey and K. Moats, Phys. Rev. D 93, 034035 (2016).

[5] D. Ebert, R. N. Faustov, and V. O. Galkin, Eur. Phys. J. C 66, 197 (2010).

[6] T. Barnes, F. E. Close, and H. J. Lipkin, Phys. Rev. D 68, 054006 (2003).

[7] E. van Beveren and G. Rupp, Phys. Rev. Lett. 91, 012003 (2003).

[8] A. P. Szczepaniak, Phys. Lett. B 567, 23 (2003).

[9] E. E. Kolomeitsev and M. F. M. Lutz, Phys. Lett. B 582, 39 (2004).

[10] Y.-Q. Chen and X.-Q. Li, Phys. Rev. Lett. 93, 232001 (2004).

[11] F.-K. Guo, P.-N. Shen, H.-C. Chiang, R.-G. Ping, and B.-S. Zou, Phys. Lett. B 641, 278 (2006).

[12] F.-K. Guo, P.-N. Shen, and H.-C. Chiang, Phys. Lett. B 647, 133 (2007).

[13] D. Gamermann, E. Oset, D. Strottman, and M. J. Vicente Vacas, Phys. Rev. D 76, 074016 (2007).

[14] H.-Y. Cheng and W.-S. Hou, Phys. Lett. B 566, 193 (2003).

[15] L. Maiani, F. Piccinini, A. D. Polosa, and V. Riquer, Phys. Rev. D 71, 014028 (2005).

[16] T. E. Browder, S. Pakvasa, and A. A. Petrov, Phys. Lett. B 578, 365 (2004).

[17] K. Abe et al. (Belle Collaboration), Phys. Rev. D 69, 112002 (2004).

[18] J. M. Link et al. (FOCUS Collaboration), Phys. Lett. B 586, 11 (2004).

[19] L. Liu, K. Orginos, F.-K. Guo, C. Hanhart, and U.-G. Meißner, Phys. Rev. D 87, 014508 (2013).

[20] D. Mohler, C. B. Lang, L. Leskovec, S. Prelovsek, and R. M. Woloshyn, Phys. Rev. Lett. 111, 222001 (2013).

[21] C. B. Lang, L. Leskovec, D. Mohler, S. Prelovsek, and R. M. Woloshyn, Phys. Rev. D 90, 034510 (2014).

[22] G. Moir, M. Peardon, S. M. Ryan, C. E. Thomas, and D. J. Wilson, J. High Energy Phys. 10 (2016) 011.

[23] G. S. Bali, S. Collins, A. Cox, and A. Schäfer, Phys. Rev. D 96, 074501 (2017).

[24] G. K. C. Cheung, C. E. Thomas, D. J. Wilson, G. Moir, M. Peardon, and S. M. Ryan, J. High Energy Phys. 02 (2021) 100.

[25] R. Aaij et al. (LHCb Collaboration), Phys. Rev. D 94, 072001 (2016).

[26] M. Albaladejo, P. Fernandez-Soler, F.-K. Guo, and J. Nieves, Phys. Lett. B 767, 465 (2017).

[27] M.-L. Du, M. Albaladejo, P. Fernández-Soler, F.-K. Guo, C. Hanhart, U.-G. Meißner, J. Nieves, and D.-L. Yao, Phys. Rev. D 98, 094018 (2018).

[28] F.-K. Guo, C. Hanhart, U.-G. Meißner, Q. Wang, Q. Zhao, and B.-S. Zou, Rev. Mod. Phys. 90, 015004 (2018).

[29] M.-L. Du, F.-K. Guo, U.-G. Meißner, and D.-L. Yao, Eur. Phys. J. C 77, 728 (2017).
[30] F.-K. Guo, C. Hanhart, and U.-G. Meißner, Eur. Phys. J. A 40, 171 (2009).

[31] Z.-H. Guo, U.-G. Meißner, and D.-L. Yao, Phys. Rev. D 92 , 094008 (2015).

[32] Z.-H. Guo, L. Liu, U.-G. Meißner, J. A. Oller, and A. Rusetsky, Eur. Phys. J. C 79, 13 (2019).

[33] X.-Y. Guo, Y. Heo, and M. F. M. Lutz, Proc. Sci., LATTICE2018 (2018) 085.

[34] X.-Y. Guo, Y. Heo, and M. F. M. Lutz, Phys. Rev. D 98, 014510 (2018).

[35] R. Aaij et al. (LHCb Collaboration), Phys. Rev. D 90, 072003 (2014).

[36] R. Aaij et al. (LHCb Collaboration), Phys. Rev. D 92, 032002 (2015).

[37] R. Aaij et al. (LHCb Collaboration), Phys. Rev. D 91, 092002 (2015); 93, 119901(E) (2016).

[38] R. Aaij et al. (LHCb Collaboration), Phys. Rev. D 92, 012012 (2015).

[39] M.-L. Du, F.-K. Guo, and U.-G. Meißner, Phys. Rev. D 99, 114002 (2019).

[40] M. Albaladejo, P. Fernandez-Soler, J. Nieves, and P. G. Ortega, Eur. Phys. J. C 78, 722 (2018).

[41] U.-G. Meißner, Symmetry 12, 981 (2020).

[42] P. A. Zyla et al. (Particle Data Group), Prog. Theor. Exp. Phys. (2020), 083C01.

[43] J. L. Rosner, Phys. Rev. D 88, 034034 (2013).

[44] F.-K. Guo, Phys. Rev. Lett. 122, 202002 (2019).

[45] W.-L. Lin and C.-C. Chiang, Lett. Nuovo Cimento Soc. Ital. Fis. 38, 503 (1983).

[46] M. J. Savage and M. B. Wise, Phys. Rev. D 39, 3346 (1989); 40, 3127(E) (1989).

[47] F. Niecknig and B. Kubis, J. High Energy Phys. 10 (2015) 142.

[48] A. P. Szczepaniak, Phys. Lett. B 747, 410 (2015).

[49] S. Gardner and U.-G. Meißner, Phys. Rev. D 65, 094004 (2002).

[50] N. N. Khuri and S. B. Treiman, Phys. Rev. 119, 1115 (1960).

[51] J. B. Bronzan and C. Kacser, Phys. Rev. 132, 2703 (1963).

[52] F. Niecknig and B. Kubis, Phys. Lett. B 780, 471 (2018).

[53] R. Omnès, Nuovo Cim. 8, 316 (1958).

[54] C. Kacser, Phys. Rev. 132, 2712 (1963).

[55] See Supplemental Material at http://link.aps.org/ supplemental/10.1103/PhysRevLett.126.192001 for technical details, parameter values from the fits and a numerical demonstration of the effect of chiral symmetry on resonance parameters extracted using an isobar model.

[56] K. M. Watson, Phys. Rev. 95, 228 (1954).

[57] W. H. Press, S. A. Teukolsky, W. T. Vetterling, and B. P. Flannery, Numerical Recipes in FORTRAN: The Art of Scientific Computing (Cambridge University Press, Cambridge, 1992).

[58] T. Mehen and R. P. Springer, Phys. Rev. D 72, 034006 (2005).

[59] P. Colangelo, F. De Fazio, F. Giannuzzi, and S. Nicotri, Phys. Rev. D 86, 054024 (2012).

[60] M. H. Alhakami, Phys. Rev. D 93, 094007 (2016).

[61] H.-Y. Cheng and F.-S. Yu, Eur. Phys. J. C 77, 668 (2017).

[62] B. Aubert et al. (BABAR Collaboration), Phys. Rev. D 79, 112004 (2009). 
[63] R. Aaij et al. (LHCb Collaboration), Phys. Rev. D 101, 032005 (2020).

[64] I. Caprini, G. Colangelo, and H. Leutwyler, Phys. Rev. Lett. 96, 132001 (2006).

[65] S. Descotes-Genon and B. Moussallam, Eur. Phys. J. C 48, 553 (2006).
[66] J. R. Peláez, Phys. Rep. 658, 1 (2016).

[67] J. R. Peláez and A. Rodas, Phys. Rev. Lett. 124, 172001 (2020).

[68] J. R. Peláez and A. Rodas, arXiv:2010.11222.

[69] L. Gayer, N. Lang, S. M. Ryan, D. Tims, C. E. Thomas, and D. J. Wilson, arXiv:2102.04973. 